\title{
Parameters Estimation in a General Failure Rate Semi-Markov Reliability Model
}

\author{
M. Fathizadeh ${ }^{1}$ and K. Khorshidian ${ }^{2}$ \\ ${ }^{1}$ Department of Statistics, Faculty of Mathematical Sciences, \\ Vali-e-Asr University of Rafsanjan, Rafsanjan, Iran \\ ${ }^{2}$ Department of Statistics, Shiraz University, Shiraz, Iran \\ m.fathi@vru.ac.ir, khorshidian@susc.ac.ir \\ Received 7 August 2012 \\ Accepted 7 July 2013
}

\begin{abstract}
A semi-Markov process with four states, has been applied for modeling two dissimilar unit cold standby systems. At the moment that operating unit fails, the standby unit is switched to operate by using a switching device that is available with unknown probability $\alpha_{1}$. It is also assumed that the failure rate of unit $i$ has the general form $h_{i}(t)=\alpha_{2 i}+\alpha_{2 i+1} t^{\beta_{i}-1}, i=1,2$, where $\alpha_{2}, \ldots, \alpha_{5}$ are non-negative unknown parameters. In favor of semi-Markov structure of the system, maximum likelihood and the Bayes estimators of the unknown parameters $\underline{\alpha}=\left(\alpha_{1}, \alpha_{2}, \ldots, \alpha_{5}\right)$ are obtained while $\beta_{i}$ are non-negative known constants. Furthermore, the estimators are obtained for systems with similar units. Finally, to compare the results a simulation study is done.
\end{abstract}

Keywords: Bayesian estimation, Cold standby systems, Maximum likelihood, Semi-Markov process

\section{Introduction}

Semi-Markov processes, nowadays, have been applied in many areas of sciences, such as reliability theory. In fact, many of the reliability systems can be modeled by semi-Markov processes, such as two dissimilar unit cold standby system, which has widely been studied and used in industry. Estimation of parameters included in reliability systems is a common job in reliability analysis. Recently, in this area, estimating the parameters of life time distributions have been received special attentions. In 1983 Sarmah and Dharmadhikari have obtained the moment estimators of the parameters included in 1-out-of-2:G repairable system when the failure and repair time distributions of the units are exponential with unknown parameters, [5]. After two decades, Sarhan and El-Gohary (2003), have estimated the parameters of this model by maximum likelihood and Bayesian methods, [4]. They showed that these methods perform better than moments estimation method. Also, the parameters of the lifetimes in k-out-of-m cold standby systems with imperfect switches have been estimated using two different approaches by Al-Ruzaiza and Sarhan, [1]. In 2004 El-Gohary studied a special case of two dissimilar unit cold standby system. He used the Markov renewal theory to estimate the unknown parameters of two similar unit cold standby systems with imperfect switches, when failure rates are linearly depend on lifetimes of the system, [3]. For detailed descriptions of semi-Markov and Markov renewal processes, see [2].

The general failure rate model, that is considered in the present paper, develops linear failure rate, exponential, Weibull and Rayleigh distribution models, as well as many other reliability systems with realistic specified life time distributions.

In this article, a semi-Markov process with four states has been considered for modeling two dissimilar unit cold standby systems. At the moment that operating unit fails, the standby unit is switched to operate by using a switching device that is available with unknown probability $\alpha_{1}$. It is also assumed that the failure rate of unit $i$ has the general form $h_{i}(t)=\alpha_{2 i}+\alpha_{2 i+1} t^{\beta_{i}-1}, i=1,2$, where $\alpha_{2}, \ldots, \alpha_{5}$ are non-negative unknown parameters. In favor of a semi-Markov structure for the system, maximum likelihood and the Bayes estimators of the unknown parameters 
$\underline{\alpha}=\left(\alpha_{1}, \alpha_{2}, \ldots, \alpha_{5}\right)$ have been obtained while $\beta_{i}$ are non-negative known constants. By the fact that the system with similar units is a special case of the studied model, the estimators have also been obtained when the system has similar units. Finally, to compare the results a simulation study has been done.

Consider a semi-Markov process with finite state space $\mathbb{E}$, also let $\mathbf{P}^{0}=\left[p_{i}^{0}: i \epsilon \mathbb{E}\right]$ be a probability distribution on $\mathbb{E}: p_{i}^{0} \geq 0, \sum_{i \in \mathbb{E}} p_{i}^{0}=1$. A Markov renewal process may be defined as follows:

Let $(\xi, \mathrm{T})=\left\{\left(\xi_{n}, T_{n}\right), n=0,1,2, \ldots\right\}$ denotes a two-dimensional stochastic process with values in $\mathbb{E} \times[0, \infty)$, then $(\xi, \mathrm{T})$ is a Markov renewal process if

1. $P\left\{\xi_{n+1}=j, T_{n+1} \leq t \mid \xi_{n}=i, T_{n}, \ldots, \xi_{0}, T_{0}\right\}=P\left\{\xi_{n+1}=j, T_{n+1} \leq t \mid \xi_{n}=i\right\}$,

2. $P\left\{\xi_{0}=i_{0}, T_{0}=0\right\}=p_{i_{0}}^{0}$.

We also assume that the probabilities in (1.1) do not depend on $n$ and denote them by

$$
\mathcal{Q}_{i j}(t)=P\left\{\xi_{n+1}=j, T_{n+1} \leq t \mid \xi_{n}=i\right\}, i, j \in \mathbb{E},
$$

as the renewal kernel, and $\mathcal{Q}(t)=\left[\mathcal{Q}_{i j}(t)\right]_{i, j \in \mathrm{E}}, t \in \mathbb{R}^{+}$, as the renewal kernel matrix. The associated counting process representing the total number of transitions within $[0, t]$ is denoted by $\{N(t): t \geq 0\}$, where $N(t)=\sup \left\{n: T_{n} \leq t\right\}$. A stochastic process $\{X(t): t \geq 0\}$ where $X(t)=\xi_{N(t)}$, is called the semi-Markov process on $\mathbb{E}$, generated by Markov renewal process with initial distribution $\mathrm{P}^{0}$ and the kernel $\mathcal{Q}(t), t \geq 0$.

\section{Description of the Model}

We will consider a mechanical system which performs by the following settings:

\subsection{Notation and assumptions}

- The system consists of two dissimilar units which operate in cold standby configuration, a switch and a repair facility.

- When operating unit fails, the standby unit is switched to operate by action of a switching device. The event that switching device performs well when required is denoted by $A$, with probability $\alpha_{1}=P(A)$.

- The system fails whether operating unit fails and the repair job has not been finished yet, or both the operating unit and the switch have been failed. In this case the whole failed system will be replaced by a new identical one.

- The failure rate of unit $i$ has the general form $h_{i}(t)=\alpha_{2 i}+\alpha_{2 i+1} t^{\beta_{i}-1}, i=1,2$, where $\alpha_{2}, \ldots, \alpha_{5}$ are nonnegative unknown parameters whereas $\beta_{i}$ are non-negative known parameters.

- The life times of operating units are non-negative random variables $V_{i}$ with distribution functions $F_{i}($.$) . The$ length of repair periods of unit $i$ is a non-negative random variable $\eta_{i}$ with distribution function $G_{i}($.$) .$ Replacing time of the failed system is a non-negative random variable $\zeta$ with distribution function $M($.$) .$

- All above random variables are mutually independent.

\subsection{The semi-Markov model}

In order to describe the semi-Markov reliability model and derive the associated renewal kernel, we will introduce the following states:

0 . the system is failed;

1. the unit 1 is operating and unit 2 is under repair;

2. the unit 2 is operating and unit 1 is under repair;

3. the unit 1 is operating and unit 2 is in standby mode.

Let $v_{0}=0, v_{1}, v_{2}, \ldots$ be the successive time instants of the system changes (state transitions). Also let the process $\{X(t), t \geq 0\}$ denotes the state of the system at time $t$, with the state space $\mathbb{E}=\{0,1,2,3\}$. Define $Z_{n}=X\left(v_{n}\right)$, 
$n=0,1,2, \ldots$. Then $\left\{\left(Z_{n}, T_{n}\right), n=0,1,2, \ldots\right\}$ is a Markov renewal process with state space $\mathbb{E}=\{0,1,2,3\}$, the associated semi-Markov process $\{X(t)\}$, and the following renewal kernels

$$
\begin{aligned}
& \mathcal{Q}_{03}(t)=M(t), \quad \mathcal{Q}_{10}(t)=F_{1}(t)-\alpha_{1} \int_{0}^{t} G_{2}(x) d F_{1}(x), \\
& \mathcal{Q}_{12}(t)=\alpha_{1} \int_{0}^{t} G_{2}(x) d F_{1}(x), \quad \mathcal{Q}_{20}(t)=F_{2}(t)-\alpha_{1} \int_{0}^{t} G_{1}(x) d F_{2}(x), \\
& \mathcal{Q}_{21}(t)=\alpha_{1} \int_{0}^{t} G_{1}(x) d F_{2}(x), \quad \mathcal{Q}_{30}(t)=\left(1-\alpha_{1}\right) F_{1}(t), \quad \mathcal{Q}_{32}(t)=\alpha_{1} F_{1}(t) .
\end{aligned}
$$

By assuming the general failure rate $h_{i}(t)$, the density functions of the life times are as:

$$
f_{i}(t)=\left(\alpha_{2 i}+\alpha_{2 i+1} t^{\beta_{i}-1}\right) \exp \left[-\left(\alpha_{2 i} t+\frac{\alpha_{2 i+1}}{\beta_{i}} t^{\beta_{i}}\right)\right] .
$$

\section{Estimation of the Parameters}

In this section we will obtain the maximum likelihood and Bayes estimators of the unknown vector $\underline{\alpha}$, based on a sequence of observations $z=\left\{\left(i_{0}, 0\right),\left(i_{1}, t_{1}\right), \ldots,\left(i_{n}, t_{n}\right)\right\}$ from the random vector $Z=\left\{\left(\xi_{0}, T_{0}\right),\left(\xi_{1}, T_{1}\right), \ldots,\left(\xi_{n}, T_{n}\right)\right\}$, as a trajectory of the semi-Markov process.

\subsection{Maximum likelihood estimation}

In favor of semi-Markov structure of the system, the maximum likelihood function becomes:

$$
\begin{aligned}
L(z ; \underline{\alpha})= & A(t) \exp \left[-\left(\alpha_{2} \tau_{2}+\alpha_{3} \tau_{3}+\alpha_{4} \tau_{4}+\alpha_{5} \tau_{5}\right)\right] \alpha_{1}^{n_{12}+n_{21}+n_{32}} \\
& \times\left(1-\alpha_{1}\right)^{n_{30}} \prod_{k \in C_{10}}\left(1-\alpha_{1} G_{2}\left(t_{k}\right)\right) \prod_{k \in C_{20}}\left(1-\alpha_{1} G_{1}\left(t_{k}\right)\right) \\
& \times \prod_{k \in C_{1}}\left(\alpha_{2}+\alpha_{3} t_{k}^{\beta_{1}-1}\right) \prod_{k \in C_{2}}\left(\alpha_{4}+\alpha_{5} t_{k}^{\beta_{2}-1}\right),
\end{aligned}
$$

where

$$
\begin{aligned}
& C_{i j}=\left\{k: i_{k-1}=i, i_{k}=j, k=1,2, \ldots, n\right\}, i, j \in \mathbb{E}, \\
& \tau_{2}=\sum_{k \in C_{1}} t_{k}, \tau_{3}=\sum_{k \in C_{1}} \frac{t_{k}^{\beta_{1}}}{\beta_{1}}, \tau_{4}=\sum_{k \in C_{2}} t_{k}, \\
& \tau_{5}=\sum_{k \in C_{2}} \frac{t_{k}^{\beta_{2}}}{\beta_{2}}, C_{1}=C_{10} \bigcup C_{12} \bigcup C_{30} \bigcup C_{32}, C_{2}=C_{20} \cup C_{21}, \\
& A(t)=\prod_{k \in C_{03}} m\left(t_{k}\right) \prod_{k \in C_{12}} G_{2}\left(t_{k}\right) \prod_{k \in C_{21}} G_{1}\left(t_{k}\right) .
\end{aligned}
$$

By taking derivatives of $\ell=\log L(z ; \underline{\alpha})$ w.r.t unknown parameters, we have

$$
\begin{aligned}
\frac{\partial \ell}{\partial \alpha_{1}} & =\frac{n_{12}+n_{21}+n_{32}}{\alpha_{1}}-\frac{n_{30}}{1-\alpha_{1}}-\sum_{k \in C_{10}} \frac{G_{2}\left(t_{k}\right)}{1-\alpha_{1} G_{2}\left(t_{k}\right)}-\sum_{k \in C_{20}} \frac{G_{1}\left(t_{k}\right)}{1-\alpha_{1} G_{1}\left(t_{k}\right)}=0, \\
\frac{\partial \ell}{\partial \alpha_{2}} & =-\tau_{2}+\sum_{k \in C_{1}} \frac{1}{\alpha_{2}+\alpha_{3} t_{k}^{\beta_{1}-1}}=0, \\
\frac{\partial \ell}{\partial \alpha_{3}} & =-\tau_{3}+\sum_{k \in C_{1}} \frac{t_{k}^{\beta_{1}-1}}{\alpha_{2}+\alpha_{3} t_{k}^{\beta_{1}-1}}=0,
\end{aligned}
$$


Fathizadeh and Khorshidian

$$
\begin{aligned}
& \frac{\partial \ell}{\partial \alpha_{4}}=-\tau_{4}+\sum_{k \in C_{2}} \frac{1}{\alpha_{4}+\alpha_{5} t_{k}^{\beta_{2}-1}}=0, \\
& \frac{\partial \ell}{\partial \alpha_{5}}=-\tau_{5}+\sum_{k \in C_{2}} \frac{t_{k}^{\beta_{2}-1}}{\alpha_{4}+\alpha_{5} t_{k}^{\beta_{2}-1}}=0 .
\end{aligned}
$$

Usually, evaluating explicit expressions for the MLE's of $\underline{\alpha}=\left(\alpha_{1}, \alpha_{2}, \ldots, \alpha_{5}\right)$, is not easy. So, numerical techniques are required to calculate the MLE's of these parameters. In the following special cases, the MLE's may be directly derived from the above system of equations:

1) $G_{1}\left(t_{k}\right)=1, G_{2}\left(t_{k}\right)=1$, as $k \epsilon C_{20}, k \epsilon C_{10}$, respectively and $\alpha_{3}=\alpha_{5}=0$; i.e., lifetimes of the units are exponential. In this case, the MLE's become $\hat{\alpha}_{1}=n_{12}+n_{21}+n_{32} / m_{1}, \hat{\alpha}_{2}=m_{2} / \tau_{2}, \hat{\alpha}_{4}=m_{3} / \tau_{4}$, where $m_{1}=n_{10}+n_{12}+n_{20}+n_{21}+n_{30}+n_{32}$ and $m_{2}, m_{3}$, are cardinal numbers of the sets $C_{1}, C_{2}$, respectively. The variance-covariance matrix of the maximum likelihood estimator, $\mathrm{V}$, may be obtained as

$$
\mathrm{V}=\left[\begin{array}{ccc}
\frac{\left(n_{12}+n_{21}+n_{32}\right)\left(n_{10}+n_{20}+n_{30}\right)}{m_{1}^{3}} & 0 & 0 \\
0 & \frac{m_{2}}{\tau_{2}^{2}} & 0 \\
0 & 0 & \frac{m_{3}}{\tau_{4}^{2}}
\end{array}\right] .
$$

2) $G_{1}\left(t_{k}\right)=1, G_{2}\left(t_{k}\right)=1$, as $k \epsilon C_{20}, k \epsilon C_{10}$, respectively and $\alpha_{2}=\alpha_{4}=0$; i.e., lifetimes of the units are Weibull with known shape parameters $\beta_{1}, \beta_{2}$. In this case, $\hat{\alpha}_{1}=n_{12}+n_{21}+n_{32} / m_{1}, \hat{\alpha}_{3}=m_{2} / \tau_{3}$, and $\hat{\alpha}_{5}=m_{3} / \tau_{5}$, also,

$$
\mathbf{V}=\left[\begin{array}{ccc}
\frac{\left(n_{12}+n_{21}+n_{32}\right)\left(n_{10}+n_{20}+n_{30}\right)}{m_{1}^{3}} & 0 & 0 \\
0 & \frac{m_{2}}{\tau_{3}^{2}} & 0 \\
0 & 0 & \frac{m_{3}}{\tau_{5}^{2}}
\end{array}\right] .
$$

Since the systems with similar units are applied more than dissimilar ones, we also check the above results for the similar case. By combining states 1 and 2, the state space reduces to the following form;

0. The system is failed;

1. One unit is operating and another is under repair;

2. One unit is operating and another is in standby mode.

By considering

$$
f(t)=\left(\alpha_{2}+\alpha_{3} t^{\beta-1}\right) \exp \left[-\left(\alpha_{2} t+\frac{\alpha_{3}}{\beta} t^{\beta}\right)\right],
$$

as the density function for the life time of units, the maximum likelihood equations become 


$$
\begin{aligned}
& \frac{\partial \ell}{\partial \alpha_{1}}=\frac{n_{11}+n_{21}}{\alpha_{1}}-\frac{n_{20}}{1-\alpha_{1}}-\sum_{k \in C} \frac{G\left(t_{k}\right)}{1-\alpha_{1} G\left(t_{k}\right)}=0, \\
& \frac{\partial \ell}{\partial \alpha_{2}}=-\tau_{2}+\sum_{k \in C} \frac{1}{\alpha_{2}+\alpha_{3} t_{k}^{\beta-1}}=0, \\
& \frac{\partial \ell}{\partial \alpha_{3}}=-\tau_{3}+\sum_{k \in C_{1}} \frac{t_{k}^{\beta-1}}{\alpha_{2}+\alpha_{3} t_{k}^{\beta-1}}=0,
\end{aligned}
$$

where, $G_{1}\left(t_{k}\right)=G_{2}\left(t_{k}\right)=G\left(t_{k}\right), \tau_{2}=\sum_{k \in C} t_{k}, \tau_{3}=\sum_{k \in C} t_{k}^{\beta} / \beta$ and $C=C_{10} \cup C_{11} \cup C_{20} \cup C_{21}$. The following results
are obtained from solving the system of equations:

1) Let $G\left(t_{k}\right)=1$, as $k \epsilon C_{10}$ and $\alpha_{3}=0$; i.e., lifetimes of the units are exponential. In this case, $\hat{\alpha}_{1}=n_{11}+n_{21} / m$, and $\hat{\alpha}_{2}=m / \tau_{2}$, where $m=n_{10}+n_{11}+n_{20}+n_{21}$. Also,

$$
V=\left[\begin{array}{cc}
\frac{\left(n_{11}+n_{21}\right)\left(n_{10}+n_{20}\right)}{m^{3}} & 0 \\
0 & \frac{m}{\tau_{2}^{2}}
\end{array}\right] .
$$

Remark 1. The results of El-Gohary [3], will be obtained by considering $\beta=2$, in the above formulas.

2) Let $G\left(t_{k}\right)=1$, as $k \epsilon C_{10}$ and $\alpha_{2}=0$; i.e., lifetimes of the system's units are Weibull with known shape parameters. In this case, $\hat{\alpha}_{1}=n_{11}+n_{21} / m$, and $\hat{\alpha}_{3}=m / \tau_{3}$. Similar to the preceding case we have

$$
V=\left[\begin{array}{cc}
\frac{\left(n_{11}+n_{21}\right)\left(n_{10}+n_{20}\right)}{m^{3}} & 0 \\
0 & \frac{m}{\tau_{3}^{2}}
\end{array}\right] .
$$

Remark 2. The formulas which have been obtained for this case, extends the results of El-Gohary [3] to the Weibull distribution which is a more general distribution.

\subsection{The Bayes estimation}

In order to obtain the Bayes estimator for the vector of unknown parameters $\underline{\alpha}=\left(\alpha_{1}, \alpha_{2}, \ldots, \alpha_{5}\right)$, the following assumptions are adopted:

A1: $\alpha_{s}(s=1,2,3,4,5)$, behave as independent random variables,

A2: $\alpha_{s}(s=1,2,3,4,5)$, has prior density function $h_{\alpha_{s}}($.$) ,$

A3: The loss function when the vector $\underline{\alpha}$ is estimated by $\underline{\hat{\alpha}}$ is quadratic.

By a calculation process similar to [3], we will arrive at our main theorem which gives the $r^{\text {th }}$ moment of the marginal posterior pdf of $\alpha_{s}(s=1,2,3,4,5)$.

Theorem 1. The $r^{\text {th }}$ moment of the marginal posterior pdf of $\alpha_{s}(s=1,2,3,4,5)$ are given by

$$
\mu_{\alpha_{s}}^{r}=\frac{\Phi\left(r \delta_{1 s}, r \delta_{2 s}, r \delta_{3 s}, r \delta_{4 s}, r \delta_{5 s}\right)}{\Phi(\underline{0})}, s=1,2,3,4,5, r=1,2, \ldots
$$

where, $\delta_{k s}$ is the Kroneker delta, and 


$$
\begin{aligned}
\Phi\left(u_{1}, u_{2}, u_{3}, u_{4}, u_{5}\right)= & \sum_{l_{1}=0 l_{2}=0 l_{3}=0 l_{4}=0 l_{5}=0 l_{6}=0 l_{7}=0 l_{8}=0}^{n_{10}} \sum^{n_{12}} \sum^{n_{20}} \sum^{n_{20}} \sum^{n_{21}} \sum^{n_{30}} \sum^{n_{32}}(-1)^{l_{1}+l_{4}} D_{l_{1} \ldots l_{8}} \\
& \times \int_{\Theta_{\alpha_{1}}} \alpha_{1}^{n_{12}+n_{21}+n_{32}+l_{1}+l_{4}+u_{1}}\left(1-\alpha_{1}\right)^{n_{30}} h_{\alpha_{1}}\left(\alpha_{1}\right) d \alpha_{1} \\
& \times \prod_{v=2}^{5} \int_{\Theta_{\alpha_{v}}} h_{\alpha_{v}}\left(\alpha_{v}\right) \alpha_{v}^{m_{2} \delta_{2 v}+\left(l_{2}+l_{3}+l_{7}+l_{8}\right)\left(\delta_{3 v}-\delta_{2 v}\right)+m_{3} \delta_{4 v}+\left(l_{5}+l_{6}\right)\left(\delta_{5 v}-\delta_{4 v}\right)+u_{v}} \times \mathrm{e}^{-\tau_{v} \alpha_{v}} d \alpha_{v},
\end{aligned}
$$

where

$$
\begin{aligned}
& \varphi_{l_{1}}=\sum_{1 \leq k_{1}<k_{2}<\ldots<k_{l_{1}} \leq n_{10}} \prod_{j=1}^{l_{1}} G_{2}\left(t_{k_{j}}\right), \quad \varphi_{l_{2}}=\sum_{1 \leq k_{1}<k_{2}<\ldots<k_{l_{2}} \leq n_{10}} \prod_{j=1}^{l_{2}} t_{k_{j}}^{\beta_{1}-1}, \quad \varphi_{l_{3}}=\sum_{1 \leq k_{1}<k_{2}<\ldots<k_{3} \leq n_{12}} \prod_{j=1}^{l_{3}} t_{k_{j}}^{\beta_{1}-1}, \\
& \varphi_{l_{4}}=\sum_{1 \leq k_{1}<k_{2}<\ldots<k_{l_{1}} \leq n_{20}} \prod_{j=1}^{l_{4}} G_{1}\left(t_{k_{j}}\right), \quad \varphi_{l_{5}}=\sum_{1 \leq k_{1}<k_{2}<\ldots<k_{5} \leq n_{20}} \prod_{j=1}^{l_{5}} t_{k_{j}}^{\beta_{2}-1}, \quad \varphi_{l_{6}}=\sum_{1 \leq k_{1}<k_{2}<\ldots<k_{l_{6}} \leq n_{21} 1} \prod_{j=1}^{l_{6}} t_{k_{j}}^{\beta_{2}-1}, \\
& \varphi_{l_{7}}=\sum_{1 \leq k_{1}<k_{2}<\ldots<k_{l_{7}} \leq n_{30}} \prod_{j=1}^{l_{7}} t_{k_{j}}^{\beta_{1}-1}, \quad \varphi_{l_{8}}=\sum_{1 \leq k_{1}<k_{2}<\ldots<k_{l_{8}} \leq n_{32}} \prod_{j=1}^{l_{8}} t_{k_{j}}^{\beta_{1}-1}, \quad D_{l_{1} \ldots l_{8}}=\prod_{i=1}^{8} \varphi_{l_{i}} .
\end{aligned}
$$

and $\Theta_{\alpha_{v}}(v=1,2,3,4,5)$ is the domain of $\alpha_{v}$.

Theorem 2. Under assumptions A1-A3, we have:

(i) The Bayes estimator for $\alpha_{s}(s=1,2,3,4,5)$ is

$$
\hat{\alpha}_{s}=\mathrm{E}\left(\alpha_{s} \mid z\right)=\frac{\Phi\left(\delta_{1 s}, \delta_{2 s}, \delta_{3 s}, \delta_{4 s}, \delta_{5 s}\right)}{\Phi(\underline{0})} .
$$

(ii) The minimum posterior risk associated to the Bayes estimator $\alpha_{s}(s=1,2,3,4,5)$ is

$$
\operatorname{var}\left(\alpha_{s} \mid z\right)=\frac{\Phi\left(2 \delta_{1 s}, 2 \delta_{2 s}, 2 \delta_{3 s}, 2 \delta_{4 s}, 2 \delta_{5 s}\right)}{\Phi(\underline{0})}-\left(\frac{\Phi\left(\delta_{1 s}, \delta_{2 s}, \delta_{3 s}, \delta_{4 s}, \delta_{5 s}\right)}{\Phi(\underline{0})}\right)^{2} .
$$

Proof. By taking $r=1,2$ in (3.1), the desired result follows immediately.

\subsubsection{Similar units}

Under the assumptions A1-A3, we can obtain the following results for the systems with similar units.

(i) The Bayes estimator for $\alpha_{s}(s=1,2,3)$ is

$$
\hat{\alpha}_{s}=\mathrm{E}\left(\alpha_{s} \mid z\right)=\frac{\Phi\left(\delta_{1 s}, \delta_{2 s}, \delta_{3 s}\right)}{\Phi(\underline{0})} \text {. }
$$

(ii) The minimum posterior risk associated to the Bayes estimator $\hat{\alpha}_{s}(s=1,2,3)$ is

$$
\operatorname{var}\left(\alpha_{s} \mid z\right)=\frac{\Phi\left(2 \delta_{1 s}, 2 \delta_{2 s}, 2 \delta_{3 s}\right)}{\Phi(\underline{0})}-\left(\frac{\Phi\left(\delta_{1 s}, \delta_{2 s}, \delta_{3 s}\right)}{\Phi(\underline{0})}\right)^{2},
$$

where 


$$
\begin{aligned}
& \Phi\left(u_{1}, u_{2}, u_{3}\right)=\sum_{l_{1}=0 l_{2}=0 l_{3}=0 l_{4}=0 l_{5}=0}^{n_{10}} \sum^{n_{10}} \sum^{n_{11}} \sum^{n_{20}} \sum^{n_{21}}(-1)^{l_{1}} D_{l_{1} \ldots l_{5}} \int_{\Theta_{\alpha_{1}}} \alpha_{1}^{n_{11}+n_{21}+l_{1}+u_{1}}\left(1-\alpha_{1}\right)^{n_{20}} h_{\alpha_{1}}\left(\alpha_{1}\right) d \alpha_{1} \\
& \times \prod_{v=2 \Theta_{\alpha_{v}}}^{3} h_{\alpha_{v}}\left(\alpha_{v}\right) \alpha_{v}{ }^{m \delta_{2 v} v\left(l_{2}+l_{3}+l_{4}+l_{s}\right)\left(\delta_{3 v}-\delta_{2 v}\right)+u_{v}} \times \mathrm{e}^{-\tau_{v} \alpha_{v}} d \alpha_{v} .
\end{aligned}
$$

\subsection{Example}

Dissimilar case: Let $\alpha_{1}$ has a Beta prior pdf

$$
h_{\alpha_{1}}\left(\alpha_{1}\right)=\frac{\alpha_{1}^{r_{1}-1}\left(1-\alpha_{1}\right)^{\lambda_{1}-1}}{\beta\left(r_{1}, \lambda_{1}\right)}, r_{1}, \lambda_{1}>0,
$$

also, let all the parameter $\alpha_{s}(s=2,3,4,5)$ has Gamma distribution

$$
h_{\alpha_{s}}\left(\alpha_{s}\right)=\frac{\lambda_{s}^{r_{s}}}{\Gamma\left(r_{s}\right)} \alpha_{s}^{r_{s}-1} \exp \left[-\lambda_{s} \alpha_{s}\right], r_{s}, \lambda_{s}>0,
$$

Then, $\Phi\left(u_{1}, u_{2}, u_{3}, u_{4}, u_{5}\right)$, become

$$
\begin{aligned}
& \Phi\left(u_{1}, u_{2}, u_{3}, u_{4}, u_{5}\right)=\frac{\prod_{v=2}^{5} \frac{\lambda_{v}^{r_{v}}}{\Gamma\left(r_{v}\right)}}{\beta\left(r_{1}, \lambda_{1}\right)} \sum_{l_{1}=0 l_{2}=0 l_{3}=0 l_{4}=0 l_{5}=0 l_{6}=0 l_{7}=0 l_{8}=0}^{n_{10}} \sum^{n_{10}} \sum_{n_{12}}^{n_{20}} \sum^{n_{20}} \sum^{n_{20}} \sum^{n_{21}} \sum_{30}^{n_{30}} \sum_{l_{1} \ldots l_{8}}^{n_{32}}(-1)^{l_{1}+l_{4}} D_{l_{1}} \\
& \times \beta\left(n_{12}+n_{21}+n_{32}+l_{1}+l_{4}+r_{1}+u_{1}, n_{30}+\lambda_{1}\right)
\end{aligned}
$$

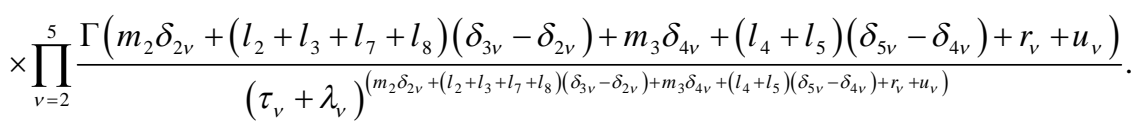

Similar case: Let $\alpha_{1}$ has a Beta prior distribution. Also let all the parameter $\alpha_{s}(s=2,3)$ has Gamma distribution. $\Phi\left(u_{1}, u_{2}, u_{3}\right)$, become

$$
\begin{aligned}
\Phi\left(u_{1}, u_{2}, u_{3}\right)= & \frac{\prod_{v=2}^{3} \frac{\lambda_{v}^{r_{v}}}{\Gamma\left(r_{v}\right)} \sum_{l_{1}=0 l_{2}=0 l_{3}=0 l_{4}=0 l_{5}=0}^{n_{10}} \sum^{n_{10}} \sum^{n_{11}} \sum^{n_{20}} \sum^{n_{21}}\left(-\lambda_{1}\right)}{\left.n_{2}\right)} D_{l_{1} \ldots l_{5}} \beta\left(n_{11}+n_{21}+l_{1}+r_{1}+u_{1}, n_{20}+\lambda_{1}\right) \\
& \times \prod_{v=2}^{3} \frac{\Gamma\left(m \delta_{2 v}+\left(l_{2}+l_{3}+l_{4}+l_{5}\right)\left(\delta_{3 v}-\delta_{2 v}\right)+r_{v}+u_{v}\right)}{\left(\tau_{v}+\lambda_{v}\right)^{\left(m \delta_{2 v}+\left(l_{2}+l_{3}+l_{4}+l_{5}\right)\left(\delta_{3 v}-\delta_{2 v}\right)+r_{v}+u_{v}\right)} .}
\end{aligned}
$$

\begin{tabular}{|c|c|c|c|c|c|c|c|c|c|c|c|}
\hline Sample & & atic & & & & & & & & & \\
\hline 1 & 2 & 0 & 2 & 1 & 1 & 1 & 0 & 2 & 1 & 1 & 0 \\
\hline 2 & 2 & 1 & 1 & 1 & 0 & 2 & 1 & 1 & 1 & 0 & 2 \\
\hline 3 & 2 & 0 & 2 & 1 & 0 & 2 & 0 & 2 & 1 & 1 & 1 \\
\hline
\end{tabular}

Remark 3. In (3.2), let $\beta=2$, then we will obtain exactly the same result of El-Gohary, [3].

\section{A Simulation Study}

In this section, we generate three samples of size 10 of semi-Markov standby model with similar units to compare the results. It is assumed that the exact values of the unknown parameters used to generate the samples are $\beta=2, \alpha_{1}=0.7, \alpha_{2}=0.5$ and $\alpha_{3}=1$.

Table 1 . Observations 
Table 2. Sojourn times

\begin{tabular}{lllllllllll}
\hline Sample & $T_{n}$ & & \multicolumn{1}{l}{} & & & & & & & \\
\hline 1 & 0.651 & 0.472 & 0.487 & 1.36 & 1.247 & 0.001 & 2.364 & 1.365 & 0.218 & 1.707 \\
\hline 2 & 0.873 & 0.007 & 1.656 & 1.17 & 1.661 & 0.563 & 0.915 & 1.819 & 2.193 & 2.199 \\
\hline 3 & 2.233 & 0.183 & 0.74 & 0.304 & 1.353 & 1.128 & 2.129 & 1.25 & 0.044 & 1.741 \\
\hline
\end{tabular}

In obtaining Bayesian estimators of unknown parameters, we assume that $\alpha_{1}, \alpha_{2}$ and $\alpha_{3}$ are random variables with prior distributions, $\operatorname{Beta}(14,6), \operatorname{Gamma}(25,50)$ and $\operatorname{Gamma}(100,100)$ respectively.

Table 3. Estimation of parameters by different methods

\begin{tabular}{|c|c|c|c|c|c|c|}
\hline \multirow[t]{2}{*}{ Sample } & \multicolumn{3}{|c|}{ Maximum likelihood method } & \multicolumn{3}{|c|}{ Bayesian method } \\
\hline & $\alpha_{1}$ & $\alpha_{2}$ & $\alpha_{3}$ & $\alpha_{1}$ & $\alpha_{2}$ & $\alpha_{3}$ \\
\hline 1 & 0.625 & 0.593 & 0.861 & 0.678 & 0.502 & 0.998 \\
\hline 2 & 0.75 & 0.323 & 0.71 & 0.714 & 0.473 & 0.979 \\
\hline 3 & 0.571 & 0.488 & 0.586 & 0.666 & 0.486 & 0.984 \\
\hline
\end{tabular}

Table 4 gives the percentages of relative errors for the estimators obtained by each method.

Table 4. Relative errors(\%)

\begin{tabular}{|c|c|c|c|c|c|c|}
\hline \multirow[t]{2}{*}{ Sample } & \multicolumn{3}{|c|}{ Maximum likelihood method } & \multicolumn{3}{|c|}{ Bayesian method } \\
\hline & $\alpha_{1}$ & $\alpha_{2}$ & $\alpha_{3}$ & $\alpha_{1}$ & $\alpha_{2}$ & $\alpha_{3}$ \\
\hline 1 & 10.714 & 18.6 & 13.9 & 3.143 & 1 & 0.2 \\
\hline 2 & 7.142 & 35.4 & 29 & 2 & 5.4 & 2.1 \\
\hline 3 & 18.428 & 2.4 & 41.4 & 4.857 & 2.8 & 1.6 \\
\hline
\end{tabular}

Results of tables 3 and 4, show that the Bayesian procedure gives better estimates than maximum likelihood method.

\section{References}

1. Al-Ruzaiza, A. S. and Sarhan, A. M., Estimators for parameters included in cold standby systems with imperfect switches, Int. J. Reliab. Appl. 6 (2005), 65-78.

2. Cinlar, E., Introduction to stochastic processes. Prentice-Hall, Inc, 1975.

3. El-Gohary, A., Bayesian estimations of parameters in a three state reliability semi-Markov models, Appl. Math. comput. 154 (2004), 53-67.

4. Sarhan, A. and El-Gohary, A., Parameter estimations of 1-out-of-2: G repairable system, Appl. Math. Comput. 145 (2003), 469-479.

5. Sarmah. P. and Dharmadhikari. A. D., Estimation of parameters of 1-out-of 2:G repairable system, Commun. Statist. Theor. Meth. 12 (14) (1983), 1609-1618. 